\title{
VARIOUS BOUNDS FOR LIAR'S DOMINATION NUMBER
}

\author{
Abdollah Alimadadi ${ }^{1}$ \\ ${ }^{1,2}$ Department of Mathematics \\ University of Tafresh, Tafresh, Iran \\ e-mail: abd.alimadadi@yahoo.com \\ Doost Ali Mojdeh ${ }^{2}$ \\ ${ }^{2}$ Department of Mathematics \\ University of Mazandaran, Babolsar, Iran \\ e-mail: damojdeh@umz.ac.ir \\ AND \\ NADER JAFARI RAD \\ Department of Mathematics \\ Shahrood University of Technology, Shahrood, Iran \\ e-mail: n.jafarirad@gmail.com
}

\begin{abstract}
Let $G=(V, E)$ be a graph. A set $S \subseteq V$ is a dominating set if $\bigcup_{v \in S} N[v]$ $=V$, where $N[v]$ is the closed neighborhood of $v$. Let $L \subseteq V$ be a dominating set, and let $v$ be a designated vertex in $V$ (an intruder vertex). Each vertex in $L \cap N[v]$ can report that $v$ is the location of the intruder, but (at most) one $x \in L \cap N[v]$ can report any $w \in N[x]$ as the intruder location or $x$ can indicate that there is no intruder in $N[x]$. A dominating set $L$ is called a liar's dominating set if every $v \in V(G)$ can be correctly identified as an intruder location under these restrictions. The minimum cardinality of a liar's dominating set is called the liar's domination number, and is denoted by $\gamma_{L R}(G)$. In this paper, we present sharp bounds for the liar's domination number in terms of the diameter, the girth and clique covering number of a graph. We present two Nordhaus-Gaddum type relations for $\gamma_{L R}(G)$, and study liar's dominating set sensitivity versus edge-connectivity. We also present various bounds for the liar's domination component number, that is, the maximum number of components over all minimum liar's dominating sets.
\end{abstract}


Keywords: liar's domination, diameter, regular graph, Nordhaus-Gaddum.

2010 Mathematics Subject Classification: 05C69.

\section{REFERENCES}

[1] D. Auger, Induced paths in twin-free graphs, Electron. J. Combin. 15 \#N17 (2008).

[2] J.A. Bondy and U.S.R. Murty, Graph Theory, Graduate Texts in Mathematics 244 (Springer-Verlag, London, 2008).

[3] G. Chartrand and L. Lesniak, Graphs and Digraphs, 4th Ed. (CRC Press, Bocz Raton, 2004).

[4] T.W. Haynes, S.T. Hedetniemi and P.J. Slater, Domination in Graphs, Advanced Topics (Marcel Dekker, Inc., New York, 1998).

[5] T.W. Haynes, S.T. Hedetniemi and P.J. Slater, Fundamentals of Domination in Graph (Marcel Dekker, Inc., New York, 1998).

[6] T.W. Haynes, P.J. Slater and C. Sterling, Liar's domination in ladders, Congr. Numer. 212 (2012) 45-56.

[7] I. Honkala, T. Laihonen and S. Ranto, On codes identifying sets of vertices in Hamming spaces, Des. Codes Cryptogr. 24 (2001) 193-204. doi:10.1023/A:1011256721935

[8] V. Junnila and T. Laihonen, Optimal identifying codes in cycles and paths, Graphs Combin. 28 (2012) 469-481. doi:10.1007/s00373-011-1058-6

[9] M.G. Karpovsky, K. Chakrabarty and L.B. Levitin, On a new class of codes for identifying vertices in graphs, IEEE Trans. Inform. Theory 44 (1998) 599-611. doi:10.1109/18.661507

[10] M. Nikodem, False alarms in fault-tolerant dominating sets in graphs, Opuscula Math. 32 (2012) 751-760. doi:10.7494/OpMath.2012.32.4.751

[11] B.S. Panda and S. Paul, Hardness results and approximation algorithm for total liar's domination in graphs, J. Comb. Optim. 27 (2014) 643-662. doi:10.1007/s10878-012-9542-3

[12] B.S. Panda and S. Paul, Liar's domination in graphs: Complexity and algorithm, Discrete Appl. Math. 161 (2013) 1085-1092.

doi:10.1016/j.dam.2012.12.011

[13] B.S. Panda and S. Paul, A linear time algorithm for liar's domination problem in proper interval graphs, Inform. Process. Lett. 113 (2013) 815-822. doi:10.1016/j.ipl.2013.07.012

[14] M.L. Roden and P.J. Slater, Liar's domination and the domination continuum, Congr. Numer. 190 (2008) 77-85. 
[15] M.L. Roden and P.J. Slater, Liar's domination in graphs, Discrete Math. 309 (2009) 5884-5890.

doi:10.1016/j.disc.2008.07.019

[16] P.J. Slater, Liar's domination, Networks 54 (2009) 70-74. doi:10.1002/net.20295

[17] J. Zhou, Z. Zhang, W. Wu and K. Xing, A greedy algorithm for the fault-tolerant connected dominating set in a general graph, J. Comb. Optim. 28 (2014) 310-319. doi:10.1007/s10878-013-9638-4

Received 11 January 2015

Revised 23 August 2015

Accepted 1 October 2015 